\title{
COMPARISON OF CONVENTIONAL AND SEROLOGICAL METHODS IN DIAGNOSIS OF CRYPTOCOCCAL MENINGITIS AMONG HIV SEROPOSITIVE INDIVIDUALS IN A SOUTH INDIAN TERTIARY CARE CENTER
}

\author{
Jayakumar Suria Kumar1, Balasubramanian Rayvathy², Chokkalingam Chandrasekar ${ }^{3}$ \\ ${ }^{1}$ Assistant Professor, Institute of Microbiology, Madurai Medical College, Madurai. \\ ${ }^{2}$ Assistant Professor, Department of Microbiology, Dr. A.L.M., PG Institute of Basic Medical Sciences, \\ University of Madras, Tharamani, Chennai. \\ 3 Professor of Pulmonary Medicine, Sri Ramachandra Medical College and Research Institute, Porur, Chennai.
}

\section{ABSTRACT}

\section{BACKGROUND}

Cryptococcal meningitis is the life threatening mycosis in HIV patients. Microscopy is poor in sensitivity and Culture is the gold standard in diagnosis. Cryptococcal Antigen detection by Latex agglutination is the rapid serological method. This study is an attempt to throw light on the simple, rapid, most sensitive and specific detection methods.

\section{AIM}

Comparative evaluation of diagnosis of Cryptococcal meningitis by using different rapid methods.

\section{METHODS}

Adult HIV positive individuals admitted at GHTM from January to December 2011, with clinical presentations suggestive of Meningitis and consenting for perform lumbar puncture were studied. From 100 cases, Cerebrospinal fluid was collected as per the SOP and subjected to India ink staining, Grams staining, Cryptococcal Ag Latex agglutination test (CAALAS) and culture. The diagnostic value was assessed by comparing the sensitivity and specificity of the three rapid methods with culture as a gold standard by using McNemar test.

\section{RESULTS}

Of the 100 tested samples, 13 and 19 were positive by India ink and Cryptococcal antigen detection respectively of which 12 samples were positive on both stains. The culture turned positive with 20 samples. Based on these reports, the sensitivity and specificity of Cryptococcal antigen detection was $95.45 \%$ and $99.45 \%$ respectively and that of India ink was $63.64 \%$ and $100 \%$ respectively.

\section{CONCLUSION}

Cryptococcal antigen detection is found superior to India ink and is a better tool for screening and confirming Cryptococcal meningitis in HIV positives. However, further and larger studies are required to recommend the use of the test in public health programmes.

\section{KEYWORDS}

HIV, Cryptococcus, Meningitis, Antigen Detection.

HOW TO CITE THIS ARTICLE: Kumar JS, Rayvathy B, Chandrasekar C. Comparison of conventional and serological methods in diagnosis of cryptococcal meningitis among HIV seropositive individuals in a South Indian tertiary care center. J. Evolution Med. Dent. Sci. 2016;5(21):1090-1092, DOI: 10.14260/jemds/2016/252

\section{INTRODUCTION}

The encapsulated yeast, Cryptococcus has rapidly risen to a worldwide highly recognizable major opportunistic fungal pathogen. Since the epidemic of HIV infections, C. neoformans has become a critically important opportunistic infection in individuals in all parts of the world who are infected with HIV. ${ }^{1}$ In immunocompromised individuals meningoencephalitis is the most common and serious clinical manifestation of $\mathrm{C}$. neoformans infection and can be refractory to antifungal therapy. ${ }^{2}$ Cryptococcal meningitis has occurred at a high frequency in India in the past two decades. ${ }^{3}$ The tropical climate of the Indian subcontinent offers a suitable

Financial or Other, Competing Interest: None.

Submission 29-01-2016, Peer Review 24-02-2016,

Acceptance 01-03-2016, Published 12-03-2016.

Corresponding Author:

Dr. Jayakumar Suria Kumar,

Assistant Professor

Institute of Microbiology,

Madurai Medical College,

Madurai-625020.

E-mail: suryjay@gmail.com

DOI: $10.14260 /$ jemds/2016/252 environment for Cryptococcus neoformans and the onslaught of the AIDS pandemic since the early 1990s has led to a sharp increase in the number of reported cases of cryptococcosis in the past decade. ${ }^{3}$

The laboratory diagnosis of C. neoformans is established by conventional methods like direct demonstration of the organism by Grams staining, Demonstration of capsule by negative staining, Cultivation of the organism, Biochemical reactions, Serological methods like demonstration of the capsular polysaccharide antigens in the Cerebrospinal Fluid (CSF) and serum and other various molecular methods.

Direct demonstration of organism by India ink staining and Grams staining are rapid and specific, but poor in their sensitivity (50-80\%). ${ }^{3}$ Culture is the "gold standard" for the diagnosis, but it is time consuming and laborious. ${ }^{4}$ Detecting antibodies is an indirect but a rapid means of diagnosis. Cryptococcal antigen detection represents the most immediate way to improve methods for Cryptococcosis serodiagnosis. ${ }^{5}$ Cryptococcal Antigen detection by Latex Agglutination Test (LAT) is a rapid and very simple method to perform. LAT is the most commonly used serological method, which is having 93\% to $100 \%$ sensitivity and $93 \%$ to $98 \%$ of specificity. 6,7 Early 
diagnosis and treatment will reduce the mortality and morbidity. Though CSF is a precious specimen, it is difficult to obtain it in large volume to perform various tests.

This study is an attempt to throw light on simple, rapid most sensitive and specific detection methods in diagnosis of cryptococcal meningitis among HIV seropositive individuals.

\section{AIMS AND OBJECTIVES}

- Comparative evaluation of diagnosis of Cryptococcal meningitis by using different rapid methods.

- To diagnose Cryptococcal meningitis in the Cerebrospinal Fluid of HIV seropositive individuals by Microscopy and Serological methods.

- To compare the Microscopy and serological results with Cryptococcal culture.

\section{MATERIALS AND METHODS}

One hundred adult HIV positive individuals belonging to both genders admitted at GHTM with clinical presentations suggestive of meningitis and consenting to perform lumbar puncture were included in this prospective study. Exclusion criteria included patients who have already been treated or are under the treatment for Cryptococcal meningitis and those with any contraindications to perform Lumbar puncture.

After obtaining informed written consent from the patient, Cerebrospinal fluid (CSF) was collected by Lumbar puncture under sterile precautions into a sterile container and the specimen was immediately transferred to the laboratory for processing. In the laboratory after the verification of request and macroscopic examination of the specimen, India ink staining, Grams staining, Cryptococcal Ag test (Latex) and culture on Sabouraud's Dextrose Agar (SDA) was performed on the same day of reception. Results were documented along with the basic demography and clinical and laboratory data of the patient. Data was analysed by using Medcalc software.

The diagnostic value of the India ink and Cryptococcal antigen detection by latex agglutination test (CAALAS Kit) was assessed by comparing the sensitivity and specificity of the two rapid methods with culture as a gold standard by using McNemar test. Ethical clearance was obtained in the Institutional Review board of our hospital.

\section{RESULTS}

Of the 100 tested samples, 13 and 19 were positive by India ink and Cryptococcal antigen detection respectively of which 12 samples were positive on both tests. The culture turned positive with 20 samples. Among the positives, 16 (80\%) were males and $4(20 \%)$ were females [Picture 1].

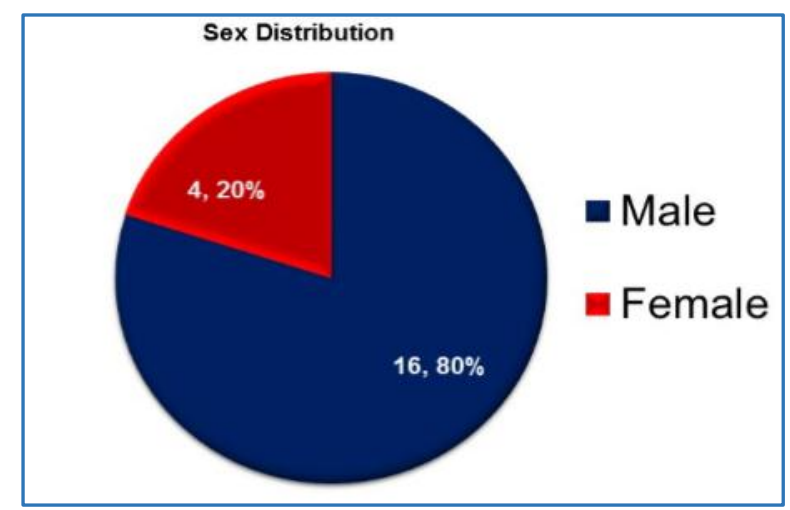

Pic. 1: Shows the Sex Distribution among the Cryptococcal Meningitis Patients

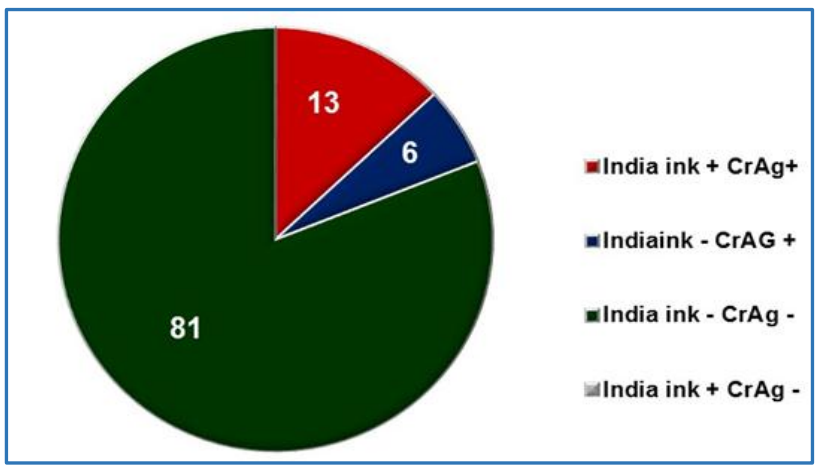

\section{Pic. 2: Overall Results by India Ink and Cryptococcal Antigen Test Methods}

As shown in Picture 2, India ink and CrAg antigen were positive in 13 samples, $\mathrm{CrAg}$ alone positive in 6 cases and none of the sample positive for India ink become Negative for $\mathrm{CrAg}$. Based on these reports, the sensitivity and specificity of Cryptococcal antigen detection was $95.45 \%$ and $99.45 \%$ respectively and that of India ink was $63.64 \%$ and $100 \%$ respectively.

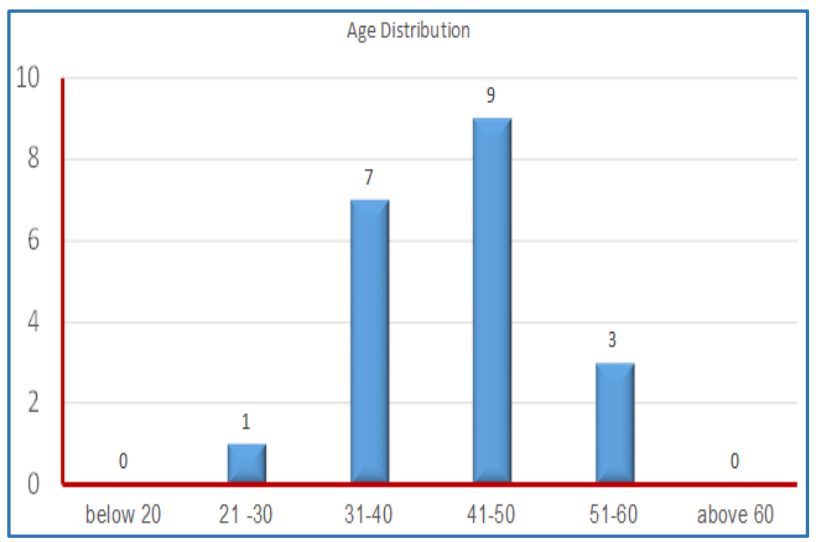

Pic. 3: Depicts the Age distribution among the Suspected Cases of Meningitis

\section{DISCUSSION}

Results of this study indicate males (Picture 1) and adults are (41-50 years) [Picture 3] are more prone to have cryptococcal meningitis. India ink is used as a screening test, but it has low sensitivity. Culture is considered as a gold standard test, but it may take up to 48 hours to grow. Cryptococcal antigen detection is rapid and simple to perform with high sensitivity and specificity as compared to India ink staining. This study results is well supported by Wake et al. $2016{ }^{6}$

\section{CONCLUSION}

On the basis of the above findings it can be said that Cryptococcal antigen detection is superior to India ink and is a better tool for screening and confirming Cryptococcal meningitis in HIV positives. Cryptococcal antigen detection by latex agglutination test is recommended by WHO as a screening and confirmatory test. However, further and larger studies are required to recommend the use of the test in public health programmes.

\section{REFERENCES}

1. Bicanic T, Harrison TS. Cryptococcal meningitis. Br Med Bull 2005;72:99-118. 
2. Banerjee U, Datta K, Majumdar T, et al. Cryptococcosis in India: the awakening of a giant? Med Mycol 2001;39:51-67.

3. Snow RM, Dismukes WE. Cryptococcal meningitis: diagnostic value of cryptococcal antigen in cerebrospinal fluid. Arch Intern Med 1975;135:1155-7.

4. Saha DC, Immaculata Xess, Neena Jain. Evaluation of conventional \& serological methods for rapid diagnosis of cryptococcosis. Indian J Med Res 2008;127:483-488.

5. Wu TC, Koo SY. Comparison of three commercial cryptococcal latex kits for detection of cryptococcal antigen. J Clin Microbiol 1983;18(5):1127-30.
6. Wake RM, Glencross DK, Sriruttan C, et al. Cryptococcal antigen screening in HIV-infected adults: let's get straight to the point. AIDS 2016;30(3):339-342.

7. Nicky Longley, Joseph Nicholas Jarvis, Graeme Meintjes, et al. Cryptococcal antigen screening in patients initiating ART in south Africa: a prospective cohort study. Clinical Infectious Diseases 2016;62(5):581-587. 